\title{
Harnessing Content and Context for Enhanced Decision Making
}

\author{
Paulo Novais, Davide Carneiro, Francisco Andrade, and José Neves \\ Department of Informatics/CCTC, University of Minho, Braga, Portugal \\ \{pjon, dcarneiro, jneves\}@di.uminho.pt \\ fandrade@direito.uminho.pt
}

\begin{abstract}
In a time in which a significant amount of interpersonal interactions take place online, one must enquire to which extent are these milieus suitable for supporting the complexity of our communication. This is especially important in more sensitive domains, such as the one of Online Dispute Resolution, in which inefficient communication environments may result in misunderstandings, poor decisions or the escalation of the conflict. The conflict manager, in particular, may find his skills severely diminished, namely in what concerns the accurate perception of the state of the parties. In this paper the development of a rich communication framework is detailed that conveys contextual information about their users, harnessed from the transparent analysis of their behaviour while communicating. Using it, the conflict manager may not only better perceive the conflict and how it affects each party but also take better contextualized decisions, closer to the ones taken in face-to-face settings.
\end{abstract}

Keywords: Online Dispute Resolution, Context-aware Computing, Stress, Fatigue

\section{Introduction}

The surroundings or the circumstances in which a given event or occurrence takes place is known as Context. It allows one to correctly understand and interpret said occurrence. Taking the field of linguistics as an example, context may refer to the set of information that is relevant to fully understand a text. This information may be very varied and include the identity of things named in the text (e.g. people, places) as well as many other aspects such as birth dates, geographical locations or temporal location. In fact, different contexts may provide completely different interpretations for the same text. Although most of the times in an unconscious way, in our daily living we constantly rely on contextual information.

The importance of Context goes as far as shaping ourselves and who we are. The knowledge acquired in the ongoing process of learning that is our life after all, comes with a strong social, cultural and physical context [2]. This bound is so strong that cognition cannot be separated from context, i.e., knowing is inseparable from activity, people, culture, language or time. 
This relationship goes as far as individuals exhibiting different cognitive and reasoning processes under different contexts: the context in which they are inserted provides the symbols and values that individuals will use [21]. Thus, no individual can be accurately and absolutely defined without a notion of context, i.e., we cannot say to another person "I behave like this" and expect to really be like that all the time. Instead, we should say "In a scenario with these conditions, I would probably behave like this".

Conflicts and their resolution, as many other processes we engage in daily, have a strong contextual background. Frequently, most of the meaning present in the underlying communication processes stems not from the words used but from accessory information that helps understanding the real meaning or purpose of the words. This includes aspects such as the body language, the gestures, the posture, the emotional response or the tone of voice.

The importance of context in conflict resolution has been noted in one of the earliest documents written about Online Dispute Resolution (ODR), by Etan Katsh [25]:

"Context can influence the approach of the neutral, the choice of process, and the behavior and attitudes of disputants. In any environment, context can affect the kinds of disputes that are likely to arise and also affect who the parties are who are likely to be involved in the dispute. Context implicitly feeds us information about the extent or nature of the injury as well as how the injury or dispute is perceived by those involved. Context situates a dispute in a particular time and place, and we react and adjust accordingly as the parameters of the environment become clear to us."

Contextual information is indeed important for the involved parties (disputants and conflict managers) to perceive the conflict in its whole, with its peculiarities, subjectivities and particular views. This paper looks at this topic, from a Computer Science perspective. It addresses different the types of context that are meaningful in the conflict resolution arena and it compares traditional ways of acquiring such information with more innovative ones, put forward by the authors in the last years. Finally, the paper briefly describes a conflict resolution platform that implements these ideas.

\section{Context-aware Computing: Harnessing Content and Context}

In the last years, context has acquired such an importance that it even gave birth to a new field in Computer Science: Context-aware Computing. It refers to systems that are aware of the state and surroundings of their users, and that are able to adapt their behaviour according to changes in their context [36]. The knowledge about the environment or the user may be relatively simple (e.g. the network to which the device is connected, the devices in the proximity) or may be more complex and even built from assumptions about the user's current situation. 
In its early days, context was much focused on the issues related to user location, encouraged mostly by the rapid emergence of mobile devices and applications who could make particular use of this information [20]. The hype revolved around applications that would provide services personalized according to their user's location. Nevertheless, in the last years the notion of context has widened significantly, and now refers not only to where the users are but also to who the users are, what the users are doing, when they are doing it and with who they are doing it. All this information allows the system to infer why the users are doing it and this is essential in the task of providing personalized, meaningful and useful services.

These novel and increasingly complex contextual models provide the support for applications that are able to adapt interfaces, improve information retrieval techniques, target services more efficiently or use implicit user-interaction techniques $[9]$.

Given the contextual richness of today's environments, information about users' context can be acquired from many different sources: from the objects the user is interacting with (e.g. title or topic of an e-book being read), inferred from the activity being performed (e.g. nature/objective of the activity), provided explicitly (e.g. social tagging, comments, bookmarks) or can be acquired from hard or soft sensors, just to name a few examples.

The potentialities of using information describing the user's context are also wide. Indeed, Many applications have already been developed that build on such notions to provide high-value and innovative services. Most of them are based on learning the patterns of the users on the environment, their preferences and their habits. This is fundamental in order for personalized services to be provided [4].

A good example of this is Magitti: an activity-aware leisure guide running on a smartphone [7]. It is essentially a recommendation service that has a model of the preferences of the user. This model is continuously built from their current situation or their past behaviours. This allows Magitti to infer the current activity of the user in order to filter and rank information items that may be of interest.

Under a different perspective, the Hearsay service developed as part of the GLOSS project [29] allows users to pick up small notes left for them in the environment. It makes sure users will find the message only in a correct context (e.g. right person in the right place at the right time). The same approach is applied to other applications, providing a structured link between environment and behaviour to improve utility and usability [19].

Other projects have also focused on acquiring contextual information from the observation of the user. [11] claim that user interactions with everyday productivity applications (e.g. word processors, Web browsers) provide rich contextual information that can be leveraged to support just-in-time access to taskrelevant information. Besides discussing the requirements for such systems and developing a general architecture, the authors present Watson, a system which gathers contextual information in the form of the text of the document the user 
is manipulating in order to proactively retrieve documents from distributed information repositories.

There is also a marked interest on the use of context-aware computing in the medical field, particularly in alleviating the tasks of medical practitioners or on supporting patients. [5] presents the design of a context-aware pill container and a context-aware hospital bed, both of which react and adapt according to what is happening in their context. The system is able to, among other tasks, point out the location of the correct medicine to take or verify if it is the correct patient receiving it.

On a different field, [34] define an innovative communication framework that incorporates Augmented Reality techniques. Users can dynamically attach newly created digital information such as voice, notes or photographs to the physical environment, through wearable computers as well as normal computers. Attached data is stored with contextual tags such as location IDs and object IDs that are obtained by wearable sensors, so the same or other wearable users can notice them when they come to the same environment. The approach implemented has a role that is similar to the one that Post-it notes play in community messaging.

A particular interest lies also on the development of context-aware systems that can be carried by the user, generally in small devices such as smartphones or video cameras. This is empowered by the functionalities and potential of current mobile devices, rich in sensors, computational power and communication capabilities. [31] address the challenge of organizing our ever-growing collections of digital photos, consequence of the enormous rise in popularity of digital cameras. To achieve it, the authors developed the MediAssist project, which uses date/time and GPS location for the organization of personal collections. The project retrieves photos of known objects (e.g. buildings, monuments) using both location information and content-based retrieval tools from the AceToolbox, allowing to improve information search and retrieval when compared to more traditional approaches.

\section{Contextualizing Conflicts}

As detailed in the previous section, Context-aware Computing has been used for many different purposes in the last years. Nonetheless, no initiatives can be found at the moment that use such approaches in the domain of conflict resolution.

After acknowledging this fact our research team, with a strong background on Ambient Intelligence, decided to tackle this challenge. In the last years we have been taking steps towards the development of a context-aware conflict resolution platform that can not only provide personalized and proactive support but also be sensitive to the state of the users. This section describes some of the approaches implemented that allow software agents and human parties to contextualize and fully understand a conflict.

In fully understanding a conflict, one of the most challenging tasks is to realistically perceive the boundaries of the case, i.e., which outcome would be realistic and relatively consensual and which outcome would be nonsense. In 
order for parties to build a realistic view on the conflict, they must be aware of a few notions such as the boundary possibilities (the best/worst possible cases) as well as the most likely outcome, according to the characteristics of the case, the norms or past experiences. In this sense, a case-based approach was developed [3] that computes the values of concepts such as the BATNA (Best Alternative to a Negotiated Agreement), WATNA (Worst Alternative to a Negotiated Agreement), MLATNA (Most Likely Alternative to a Negotiated Agreement) or the ZOPA (Zone of Possible Agreement) [30, 22, 39]. With these values parties can realistically frame their conflict and understand what their possibilities are.

Nonetheless, one of the best ways for someone to understand a present event that is taking place or going to take place in the future, is to analyse a similar event that took place in the past, under a similar context. In Computer Science, the field of Case-based Reasoning deals with approaches that are based on this notion. In this field, a case represents a past experience, teaching a past lesson, properly contextualized [26]. Depending on the domain of application, a case should contain a description of the state of the world when the event occurred (i.e. the problem description), the derived solution for the problem and/or the achieved outcome (i.e. the description of the state of the world after the case occurred) [18]. The individual can analyse the past event, with all its characteristics and within its contextual framework to fully understand it. Then, he will be in a better position to understand a similar event that may occur in the future.

The analysis of past cases is also of interest in the domain of conflict resolution. Disputant parties involved in a conflict resolution process may leverage on past cases, under a similar context, to better perceive the present. This allows the parties to calmly understand the important aspects of the past cases, being better prepared to fully understand their own and gaining a more realistic and objective view on the case. For example, if a given party is too greedy but notices that the outcome he desires has never happened in the past, he may conclude that such outcome is unlikely and even unrealistic. He may then reconsider his objectives and go for a more middle-ground solution. In order to achieve such objectives, case-retrieval approaches have been implemented by our research team that provide parties with an intuitive way to perceive their conflict and their chances in the conflict through the analysis of past similar cases [12].

Another very important aspect when contextualizing a conflict is the personal conflict handling style of each individual: it determines the expected behaviour of an individual before a conflict. Each individual has their own style, which may however vary according to variables such as the identity of the other parties, the nature of the conflict, or even the level of escalation of the conflict. Knowing the conflict handling style of the parties is especially important for the mediator, who can better prepare the strategy for the resolution of the conflict. Gathering and providing evidence about the party's conflict handling style may also be used by the mediator as a way to put pressure and to lead the parties into changing an undesired behaviour: a mediator may show to a party that he is 
being an obstacle to the successful resolution of the conflict by being too greedy or competitive. We have addressed this issue by devising an approach to infer the conflict handling style without the use of the traditional questionnaires. We rather look at the proposals exchanged by the parties during the resolution of the conflict and, framed in the boundaries of the case, build a notion of how greedy, cooperative or benevolent the parties are [3]. All this is done in real-time and in a non-invasive way, providing the mediator with very important contextual information about the case and the parties.

In seeking to contextualize a conflict an its setting, the level of escalation should not be forgotten. It describes how confrontational, violent, painful or "less comfortable" a conflict is. Desirably, parties involved in a conflict are in a cooperative and compliant state. However, in general this is not the case. If not adequately managed, the conflict may escalate to increasingly worse states, in which individuals passively or actively resist to proposals or commands, with the aggravating of the escalation potentially resulting in violence. Therefore, it is mandatory that the conflict manager is able to perceive signs of escalation of the conflict. Typically he can do so through cues such as the tone of voice, signs of inflammatory speech, gestures, body postures or the use of particular words. It is the responsibility of the mediator not only perceives these signs but to act in order to prevent the conflict to escalate further. Nonetheless, this may result particularly difficult in an online setting, as the mediator lacks the non-verbal cues that show how a person is feeling. Thus, alternatives should be devised that can inform the mediator of the level of escalation of the conflict when he is not meeting the parties face-to-face.

One of the possible approaches, prototyped by our research team in the last two years, does so by measuring the level of stress of the participants. Stress is a universal phenomenon that affects virtually our whole life. Low levels of stress make us soft, depressive and with lack of motivation, while continued high levels of stress may result in exhaustion and breakdowns [14]. The resolution of a conflict may be a particularly stressful process for many reasons, including the potential emotional charge (mostly when the parties had a prior relationship that may affect the process), the significant amount of gains or losses involved, the fear of the novelty or the unknown, mostly when parties engage in such a process for the first time. In the short-term, high levels of stress lead to cloudedmind, poor decision-making, irritability, lack of judgement or violence. Failing to control these effects may jeopardize the whole conflict resolution process and even the relationship among the parties involved.

It is thus important that the mediator is able to perceive signs of stress evidenced by the parties, and act accordingly in order to mitigate its effects by performing the necessary changes in the conflict resolution process. In this sense, an approach was developed by our research team that quantifies the level of stress of an individual from the analysis of their interaction patterns with common technological devices such as smartphones or computers. It is a noninvasive and non-intrusive approach that does not rely on questionnaires but on 
the sheer observation of the individual's behaviours [14]. Further details on this approach are given further ahead.

A similar approach is used to quantify the level of fatigue of the individuals [32]. Fatigue is a particular feeling of tiredness in which individuals experience lack of energy, lethargy or languidness. Two main forms of fatigue can be identified: physical fatigue, which defines a temporary physical inability of a muscle to function normally, and mental fatigue, which is the temporary inability to maintain optimal cognitive performance. Both types of fatigue have their negative consequences. However, in the context of a conflict resolution process, mental fatigue may be more disturbing.

Mental fatigue may be caused by sleep deprivation, long periods of work, mental stress or over-stimulation, among others. A mentally fatigued individual will have a reduced cognitive capacity, namely in terms of memory, attention and decision making. A fatigued individual participating in a conflict resolution process may take poor decisions, be unable to keep up with the process or even become irritable and uncooperative. It is therefore important that the mediator is able to detect early signs of fatigue through the behaviours and attitudes of the parties and act accordingly, namely by making pauses or by rescheduling the continuation of the process. Once again, the mediator is responsible for detecting such signs and warn the parties or take actions that prevent them from taking bad decisions, such as making a pause or resuming on another time. On the other hand, parties themselves could profit from some kind of notification, provided directly by the platform, letting them know that they may not be in their best shape to take binding or relevant decisions.

To sum up, in order to properly contextualize a conflict and its resolution, we look at its important boundary values, at past similar cases, at the conflict handling style of the parties and at the levels of stress and fatigue of these same parties. All of this is incorporated into a single conflict resolution platform, designated UMCourt. Moreover, all this information is compiled in a non-invasive and non-intrusive way, in line with the Context-aware Computing philosophy. While some of these aspects have already been addressed in detail in the past, the quantification of stress and fatigue, by being the most recent, innovative and interesting, are detailed further ahead in this paper.

\section{Traditional Approaches on Context Acquisition}

Stress, fatigue or emotional state are extremely important in describing the inner state of an individual, which ultimately affects all their conscious and unconscious actions and decisions. These personal, subjective and conscious experiences have known effects at the level of psycho-physiological expressions, biological reactions, mental states, mood, temperament, disposition, personality, motivation and, ultimately, health and well-being. Hence the significance of their study, which is very complex, involving fields such as psychology, philosophy, neurology, physiology, or medicine. 
Traditionally, two main approaches may be followed to study such phenomena. The field of psychology relies more on the use of questionnaires or surveys, whereas the field of medicine relies on different kinds of sensors. Each of these approaches has advantages and disadvantages of their own.

Surveys are generally seen as a cost-effective way of gathering large amounts of information. They do not require much effort from the researcher and often have standardized answers that make it simple to compile data [1]. They are eminently practical and may be carried out by the researcher or by any other person without significantly affecting its validity and reliability. However, surveys also suffer from a number of problems that go beyond the traditional ones related to question constructing and wording [33]. Namely, surveys are particularly inadequate to understand some complex issues such as emotions, behaviours or feelings. They are based on the individual's perception of rather subjective perceptions such as good, poor, high or low. People can also hide information, lie voluntarily, or unconsciously depreciate/overvalue certain signs [28]. It is nearly impossible for the researcher to detect such behaviours. Finally, when developing the questionnaire or survey, researchers make their own decisions and assumptions as to what is or is not important. Even if the individual finds some aspect of importance, they may not express it if it is not mentioned in the questionnaire or if it is not mentioned appropriately.

The medical field developed a highly accurate approach on the problem, based on a wide range of different sensors that measure changes on physiological or neurological features of the human body, affected by fatigue, stress or emotions. Currently, one of the most accurate indicators in use is cortisol [38], measured in the saliva, hair or blood. It is particularly useful to measure the level of stress of human beings, since this hormone is released in response to this symptom.

Other approaches on the problem may also be followed using other sensors or combinations of sensors. The Galvanic Skin Response measures the electrical conductance of the skin, which varies with its moisture level. This is of interest since the sweat glands are controlled by the sympathetic nervous system, so skin conductance is used as an indication of psychological or physiological arousal, which may happen due to stress or fatigue. The temperature of the skin, the hearth rate, or the respiratory rate are also key indicators for the study of stress or emotions $[6,23,24]$. In particular, hearth rate variability, the physiological phenomenon of variation in the time interval between heartbeats [10], has been used increasingly to study stress as it is highly related to it [8].

The significant emergence of biofeedback tools in the last years is also noteworthy. They provide combined feedback about many of the body's functions, using instruments that analyse brainwaves, muscle tone, skin conductance, heart rate or pain perception [37]. The study of brainwaves is particularly interesting as it provides clues about the inner state of an individual, in aspects such as fatigue, stress level, arousal or emotional state. Additionally, biofeedback tools can be used to improve certain aspects or habits of the daily living, as they allow perceiving changes in the body and mind affected by such habits [27]. 
In general, such sensor based-approaches can be deemed highly accurate and are used not only to assess the state of individuals, but also as a base to perform medical treatments and interventions. Their use is thus unquestionable and unparalleled in the medical arena.

Nevertheless, in the context of this paper we must look at both approaches, questionnaires and physiological sensors, from the point of view of someone who intends to build a context-aware conflict resolution platform. Thus, one must ask to which extent are questionnaires or physiological sensors suitable approaches to assess the user's state in a Virtual Environment. We argue that they do not constitute suitable approaches. Let us look at both approaches and their potential disadvantages.

When individuals use questionnaires to describe themselves and their behaviour, they sometimes do not fit in any of the four answers of the multiple choice question. They may choose not to answer, or select the option they may think to be closest to what they would do. Moreover, they have doubts quantifying some of the other answers. While some of the concepts used, such as never or always, are easy to understand, others such as often or occasionally are unclear. Additionally, the individuals that undertake this process end up behaving differently when the process is under its way. Indeed, they may assume that they are going to act in a given way while they are filling in the questionnaire, but, under the pressure of the proceedings, individuals may behave in a different way.

When the same individuals use physiological sensors, they are not comfortable or are even refusing to use sensors to which they are connected constantly, seriously limiting their movements. Moreover, they may not be entirely sure about which information will be collected and what it will be used for. Hence, they are reluctant and the sheer use of such devices seems to stress them and to deflect their attention from the conflict resolution itself.

It results clear that none of these approaches looks reasonable to quantify the state of individuals in a conflict resolution process conducted online. Hence, the following sections depict a new paradigm in which the behaviour of the individuals is analysed in order to infer information about their inner state. Indeed, phenomena such as stress, fatigue or emotions affect not only our physiology but also our behaviours. If we have a way of identifying and measuring behaviours, and if we have a way to relate given behaviours to given states, we may be able to infer the inner state of an individual through the observation of their conduct.

\section{Acquiring Contextual Features from Behavioural Analysis}

The study of stress or fatigue, including their causes and symptoms, has been a topic of disciplines such as Medicine or Psychology. Traditionally, data about users is acquired either through self-reporting mechanisms (generally questionnaires), or through the use of physiological sensors. As seen above, both have disadvantages of their own. 
In that sense, this section puts forward a new approach based on behavioural analysis. The key idea is to observe, in a non-invasive way, the behaviour of the individuals, and map certain known behaviours to specific states or changes in these states. This approach can thus be put beneath the umbrella of Behavioral Biometrics [40], and results in a multi-modal approach on the problem of behavioural analysis, where individuals' symptoms are taken as input. Such approaches yield accuracy rates that exceed their unimodal counterparts [17].

Specifically, we target behaviours that can be observed commonly in a typical scenario of use of an ODR platform without the need to use additional or invasive sensors. In that sense, we consider aspects such as the movement patterns of the user in the environment and the patterns of interaction with devices such as computers (through the mouse and the keyboard), smartphones or touch screens. From these devices, a wide range of features can be extracted that characterize behaviours that, as our previous studies conclude, are significantly affected by stress [14],[32].

The following features are extracted from the mentioned devices:

- Touch pattern - this information is acquired from touch screens with support for touch intensity. It represents the way the pressure changes over time, during a touch;

- Touch accuracy - the relationship between touches in active controls versus touches in passive areas (e.g. areas without controls, empty areas), where touches are pointless. This information is acquired from touch screens;

- Touch intensity - the amount of pressure exerted by the finger on the touch screen. It is analysed in terms of the maximum, minimum and average intensity of each touch;

- Touch duration - the time span between the beginning and the end of the touch event. This data is acquired from devices with touch screens;

- Amount of movement - its evaluation is provided by the INT3-horus framework. The image-processing stack uses the principles established by [15], and uses image difference techniques to evaluate the amount of movement between two consecutive frames [16];

- Acceleration - the acceleration is measured from accelerometers integrated or fitted into the mobile devices, the keyboard or the mouse;

- Score - this feature quantifies how well the individual performs on the several tasks he was assigned;

- Stressed touches - this feature describes which touches are classified as stressed, according to the shape of the intensity curve;

- Key Down Time - the timespan between the pressing down and the release of a key, i.e., for how long was a given key pressed;

- Time Between Keys - how long did the individual take to press another key after the previous one was released;

- Mouse Velocity - the distance travelled by the mouse (in pixels) over the time (in milliseconds);

- Mouse Acceleration - the increase in velocity of the mouse (in pixels/milliseconds) over the time (in milliseconds); 
- Time Between Clicks - the time span between each two consecutive clicks;

- Double Click Duration - the duration of a double click event, whenever this time span is inferior to 200 milliseconds. Wider time spans are not considered as double clicks;

- Average Excess of Distance - the excess of distance, in average, that the pointer travels between each two consecutive clicks of the mouse, when compared to the straight line between the same points which represents the shortest (more efficient) path;

- Average Distance of the Mouse to the Straight Line - it measures the average distance of the mouse, between each two consecutive clicks, to the straight line defined by the two consecutive clicks;

- Distance of the Mouse to the Straight Line - this feature is similar to the previous one. However, it returns the total of the distance travelled by the mouse rather than a computed average of the mouse's trajectory;

- Signed Sum of Angles - here the aim is to determine how much the movement of the mouse turns more to the right or to the left;

- Absolute Sum of Angles - this feature is quite similar to the previous one. However, it seeks to find how much the mouse turned in absolute terms, i.e., without considering the direction;

- Distance Between Clicks - it stands for the distance travelled by the mouse (in pixels) between each two consecutive clicks.

These features ensure two things. First of all, their number and different sources increase the availability of sources of data at all times, i.e., the user may stop interacting with the computer but start interacting with the smartphone, and we can acquire meaningful data to characterize their context nonetheless. Secondly, these features provide insights into different modalities affected by stress, namely the physical (through the movement pattern for example), the behavioural (through changes in the typing rhythm) or the cognitive (through measures of score). Its multi-modal nature provides this approach with higher accuracy than uni-modal ones [17].

In the last two years we have been using this approach to study and understand how stress and fatigue influence our interaction and our behaviour in such environments. Essentially, under small periods of acute stress people become more efficient and perform their tasks quickly and more efficiently. This efficiency tends to drop significantly after some time, depending on the intensity of the stressors. Fatigue, on the other hand, starts to become noticeable through a decrease in the performance and a generalized slowness in the interaction with the devices (e.g. slower mouse velocity, larger keydown time) [14], [32], [35]. These findings are not surprising nor exceptionally revealing. Indeed, the importance of this study lies not on such conclusions but on the datasets and models trained that depict how people behave when under certain conditions, that can be used to build an environment that is sensitive to its users' state, in real time.

This classification, in real time, can be crucial for the mediator to accurately understand the state of the parties and take appropriate actions. If the mediator 
notices that one of the parties is showing significant signs of fatigue, they may advise that party to make a pause, go for a walk or they may even decide to resume the process on the following day. Moreover, a fast and significant change in the level of stress may be indicative of a sudden escalation of the conflict. In this scenario, the mediator may decide to calm down the parties by making a pause, changing the subject or even interrupt the direct contact between the parties. To make this kind of decisions, the mediator may take into consideration raw data and an explanation of its meaning. Figure 1 , for example, depicts a steady increase in the keydown time (from $80 \mathrm{~ms}$ to $100 \mathrm{~ms}$ ) in the period of a few hours, a clear and known sign of increasing fatigue.

Nonetheless, the potentially large amount of information to consider in each instant and the complex inter-relation between the several sources of information may make it difficult for the mediator to draw the correct conclusions in due time. Moreover, it may overload the mediator with additional tasks and shift their focus of attention from the conflict. In that sense, more refined interfaces are available that provide the high-level knowledge compiled in each instant (Figure 2). This makes it easier for the mediator to perceive the state of the parties. Nonetheless, the mediator still has the possibility to access the more detailed data or even the raw data depicted in Figure 2 , whenever they fill it is necessary for a better perception of the state of the parties.

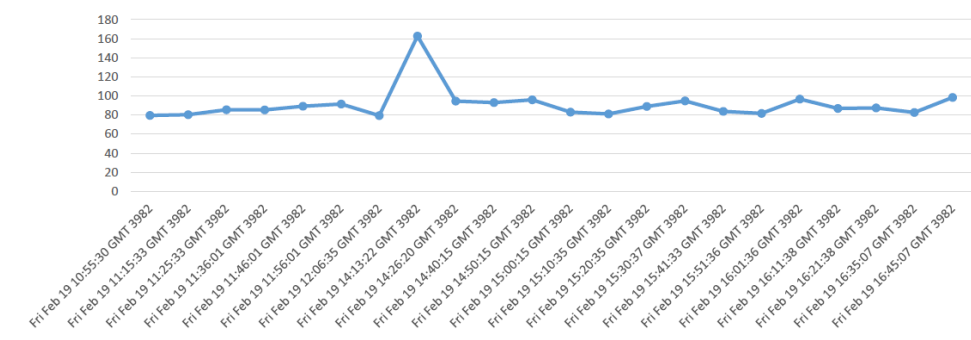

Fig. 1. Evolution of the keydown time for a user during a few hours of the day: it increases steadily as fatigue settles in.

\section{Conclusion}

Communication tools are turning our daily interactions colder and dehumanized. Our communication process, generally very rich in accessory information, is being significantly simplified to rely on words alone. This leaves aside very important contextual information such as the tone of our voice, the rhythm of speech, our gestures, our body language or our facial expressions. Indeed, many of the times, this contextual information contains more meaning than words themselves. This can be particularly worrying in a domain such as the one of 


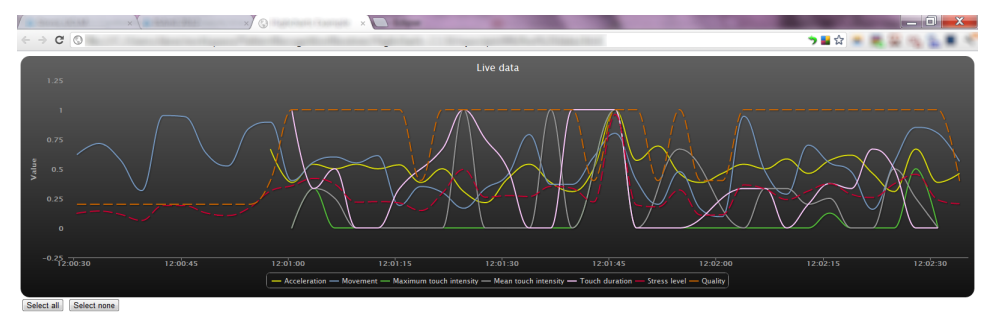

Fig. 2. Evolution of the level of stress of a user through time (dashed red line), the quality of the information (dashed orange line) and the different features available and their contribution to the level of stress.

conflict resolution, in which very important decisions are taken based on the communication between the parties.

Mediators and disputant parties must take important decisions "in the dark", without having access to the whole gamut of information that would be available in a face-to-face setting. In that sense, we described a conflict resolution framework that has been under development in the last years. Apart from the more traditional services developed in the past (e.g. information retrieval, generation of solutions), we have now been focusing on improving the communication layer of the framework, by including contextual information that is meaningful in a conflict resolution process.

Information and Communication technologies, that have been deemed to bring people closer together, seem to do the exact opposite in some aspects. It is our conviction that approaches such as the one described in this paper, focused on sensing and perceiving the state of the parties, will result in online tools for conflict resolution that actually bring the parties closer to each other, in a human sense rather than in purely practical one.

\section{Acknowledgements}

This work is part-funded by ERDF - European Regional Development Fund through the COMPETE Programme (operational programme for competitiveness) and by National Funds through the FCT - Fundação para a Ciência e a Tecnologia (Portuguese Foundation for Science and Technology) within project FCOMP-01-0124-FEDER-028980 (PTDC/EEI-SII/1386/2012) and project PEstOE/EEI/UI0752/2014.

\section{References}

1. Ackroyd, S., Hughes, J.A.: Data Collection in Context. Longman (1981)

2. Anderson, J.R., Reder, L.M., Simon, H.A.: Situated learning and education. Educational Researcher 25 (4): 5-11 (1996) 
3. Andrade F., Novais P., Carneiro D., Zeleznikow J., Neves J.: Using BATNAs and WATNAs in Online Dispute Resolution, in New Frontiers in Artificial Intelligence (JSAI-isAI 2009 Workshops, LENLS, JURISIN, KCSD, LLLL, Tokyo, Japan, 2009, Revised Selected Papers), Springer - LNAI 6284, pp. 5-18 (2010)

4. Aztiria, A., Izaguirre, A., Augusto, J.C.: Learning patterns in ambient intelligence environments: a survey. Artificial Intelligence Review, June 2010, Volume 34, Issue 1, pp. 35-51 (2010)

5. Bardram, J.E.: Applications of context-aware computing in hospital work: examples and design principles. SAC '04 Proceedings of the 2004 ACM symposium on Applied computing, pp. 1574-1579, ACM New York, NY, USA (2004)

6. Barreto, A., Zhai, J., Adjouadi, M.: Non-intrusive Physiological Monitoring for Automated Stress Detection in Human-Computer Interaction. M. Lew et al. (Eds.): HCI 2007, LNCS 4796, pp. 29-38 (2007)

7. Bellotti, V.: The Magitti activity-aware leisure guide. Social Brain Forum, Tokyo Institute of Technology, Tokyo, Japan (2008)

8. Bernardi, L., Wdowczyk-Szulc, J., Valenti, C., Castoldi, S., Passino, C., Spadacini, G., Sleight, P.: Effects of controlled breathing, mental activity and mental stress with or without verbalization on heart rate variability. Journal of the American College of Cardiology, 35(6), 1462-1469 (2000)

9. Bolchini, C., Curino, C.A., Quintarelli, E., Schreiber, F.A., Tanca, L.: A data-oriented survey of context models. ACM 36 (4): 19-26. doi:10.1145/1361348.1361353. ISSN 0163-5808 (2007)

10. Brüser, C., Stadlthanner, K., de Waele, S., Leonhardt, S.: Adaptive Beat-to-Beat Heart Rate Estimation in Ballistocardiograms. IEEE Transactions on Information Technology in Biomedicine 15 (5): 778-786. doi:10.1109/TITB.2011.2128337 (2011)

11. Budzik, J., Hammond, K.J.: IUI '00 Proceedings of the 5th international conference on Intelligent user interfaces, pp. 44-51, ACM New York, NY, USA (2000)

12. Carneiro, D., Novais, P., Andrade, F., Zeleznikow, J., Neves, J.: Using Case-based Reasoning to Support Alternative Dispute Resolution, in Distributed Computing and Artificial Intelligence, Springer - Series Advances in Intelligent and Soft Computing, vol. 79, ISBN: 978-3-642-14882-8, pp. 123-130 (2010)

13. Carneiro, D., Gomes, M., Novais, P., Andrade, F., Neves, J: Automatic Classification of Personal Conflict Styles in Conflict Resolution. IOS Press - Frontiers in Artificial Intelligence and Applications, Vol. 235, ISBN 978-1-60750-980-6, pp 43-52 (2011)

14. Carneiro, D., Carlos, C.J., Novais, P., Fernández-Caballero, A., Neves, J.: Multimodal Behavioural Analysis for Non-invasive Stress Detection, Expert Systems with Applications, Volume 39, Issue 18, December, pp. 13376-13389, Elsevier (2012)

15. Castillo, J.C., Rivas-Casado, A., Fernández-Caballero, A., López, M.T., MartínezTomás, R. A multisensory monitoring and interpretation framework based on the model-view-controller paradigm. In: Proceedings of the 4th International Workshop on the Interplay between Natural and Artificial Computation, vol 1, pp. 441-450 (2011)

16. Fernández-Caballero, A., Castillo, J.C., Martínez-Cantos, J., Martínez-Tomás, R. Optical flow or image subtraction in human detection from infrared camera on mobile robot. Robotics and Autonomous Systems 58 (12), pp. 1273-1281 (2010)

17. D'Mello, S., Kory, J.: Consistent but modest: a meta-analysis on unimodal and multimodal affect detection accuracies from 30 studies. In: Proceedings of the 14 th ACM international conference on Multimodal interaction. pp. 31-38. ICMI '12, ACM, New York, NY, USA (2012) 
18. David, B.S.: Principles for case representation in a case-based aiding system for lesson planning. In: Proceedings of the Workshop on Case-Based Reasoning, Washington (1991)

19. Dearle, A., Kirby, G.N.C., Morrison, R., McCarthy, A., Mullen, K., Yang, Y., Connor, R.C.H., Welen, P., Wilson, A.: Architectural Support for Global Smart Spaces. MDM '03 Proceedings of the 4th International Conference on Mobile Data Management, pp. 153-164, Springer-Verlag London, UK, ISBN: 3-540-00393-2 (2003)

20. Dey, A. K.: Understanding and Using Context. Personal Ubiquitous Computing 5 (1): 4-7. doi:10.1007/s007790170019 (2001)

21. Eysenck, M.W., Keane, M.T.: Psychology Press, first edition. ISBN 978$1841693590(2005)$

22. Fisher, R., Ury, W.: Getting To Yes: Negotiating Agreement Without Giving In. Boston: Houghton Mifflin (1981)

23. Healey, J.A., Picard, R.W.: Detecting stress during real-world driving tasks using physiological sensors. IEEE Transactions on Intelligent Transportation Systems, Volume 6, Issue 2 (2005)

24. Jovanov, E., O’Donnell Lords, A., Raskovic, D., Cox, P.G., Adhami, R., Andrasik, F.: Engineering in Medicine and Biology Magazine, IEEE, Volume 22 , Issue 3, pp. 49-55 (2003)

25. Katsh, E.: The Online Ombuds Office: Adapting Dispute Resolution to Cyberspace. http://www.umass.edu/dispute/ncair/katsh.htm. Last accessed in January, 2013.

26. Kolodner, J. L.: Case-based Reasoning. Morgan Kaufmann Publishers (1993)

27. Lubar, J. F.: Discourse on the development of EEG diagnostics and biofeedback for attention-deficit/hyperactivity disorders. Biofeedback and Self-regulation, 16(3), pp. 201-225 (1991)

28. Milne, J.: Questionnaires: advantages and disadvantages. Evaluation cookbook (1999)

29. Munro, A., Welen, P., Wilson, A.: Interaction Archetypes. GLOSS Consortium Report D4 (2001)

30. Notini, J.: Effective Alternatives Analysis In Mediation: "BATNA/WATNA" Analysis Demystified, (http://www.mediate.com/articles/notini1.cfm) (2005). Last accessed November 2013.

31. O'Hare, N., Gurrin, C., Jones, G.J.F., Smeaton, A.F.: Combination of content analysis and context features for digital photograph retrieval. 2nd European Workshop on the Integration of Knowledge, Semantics and Digital Media Technology (EWIMT 2005), pp. 323-328 (2005)

32. Pimenta, A., Carneiro, D., Novais, P., Neves, J.: Monitoring Mental Fatigue through the Analysis of Keyboard and Mouse Interaction Patterns. In Hybrid Artificial Intelligent Systems (pp. 222-231). Springer Berlin Heidelberg (2013)

33. Popper, K.: The Logic of Scientific Discovery, reprinted (2004) by Routledge, Taylor \& Francis (1959)

34. Rekimoto, J., Ayatsuka, Y., Hayashi, K.: Wearable Computers. Digest of Papers. Second International Symposium on. DOI 10.1109/ISWC.1998.729531 (1998)

35. Rodrigues, M., Gonçalves, S., Carneiro, D., Novais, P., Fdez-Riverola F.: Keystrokes and Clicks: Measuring Stress on E-learning Students. Management Intelligent Systems - Advances in Intelligent Systems and Computing Volume 220, 2013, pp 119-126 (2012)

36. Satyanarayanan, M.: IEEE Pervasive Computing: From the Editor in Chief - Challenges in Implementing a Context-Aware System. IEEE Distributed Systems Online 3(9) (2002) 
37. Schwartz, M. S., Andrasik, F. E.: Biofeedback: A practitioner's guide. Guilford Press (2003)

38. Staufenbiel, S.M., Penninx., B.W.J.H., Spijker , A.T., Elzinga, B.M., van Rossum E.F.C.: Hair cortisol, stress exposure, and mental health in humans: A systematic review, Psychoneuroendocrinology, Volume 38, Issue 8, August 2013, Pages 12201235, ISSN 0306-4530 (2013)

39. Steenbergen, W.: Rationalizing Dispute Resolution: From best alternative to the most likely one, in Proceedings 3rd ODR workshop, Brussels (2005)

40. Yampolskiy, R.V., Govindaraju, V.: Behavioural biometrics: a survey and classification. International Journal of Biometrics, Volume 1, Issue 1, June 2008, pp. 81-113 (2008) 